\title{
Identity-Based Multi-Signcryption with Public Verifiability
}

\author{
YU Xiu-ying ${ }^{1, a}$, He Da-ke ${ }^{2, b}$ \\ 1.School of Information Since \& Technology ,South West Jiao Tong University, 610031, China \\ 2. School of Information Science and Technology, South West Jiao Tong University,610031, China \\ aniniyxy@163.com, bdkhe_scce@swjtu.edu.cnl
}

Key words: signcryption ; identity-based; bilinear pairing; public verifiability

\begin{abstract}
Multi-signcryption can meet the requirement of message signcryption with muti-participant. Since the existing identity-based multi-signcryption scheme cannot offer the function of public verifiability, based on identity and bilinear pairing on the Elliptic Curve, a new scheme with public verifiability is proposed. In the scheme, with the steps which is comparatively independent to the signcryption process, it can provide the public verification of each signcryption in need. Therefore, our scheme efficiently achieves the cryptographic functions of multi-signcryption.
\end{abstract}

\section{Introduction}

Identity based cryptosystems were introduced by Shamir in1984 ([1]). The idea was to get rid of public key certicates by allowing the user's public key to be the binary sequence corresponding to an information identifying him in a non ambiguous way (e-mail address, IP address combined to a user name, social security number,...).Since Boneh and Franklin gave a practical ID-Based encryption scheme [2] from Weil pairing in 2001, a large number of papers have been published in this area.The concept of public key signcryption schemes was found by Zheng in 1997 ([3]). The idea of this kind of primitive is to perform encryption and signature in a single logical step in order to obtain confidentiality, integrity, authentication and non-repudiation more efficiently than the sign-then-encrypt approach. Many schemes have been designed as the extension of signcryption such as proxy signcryption, Multi-proxy-signcryption, ID-based signcryption,ect, and many research have been proposed[4-8].

With the continue growth of the internet, user sends and forwards an original message to other users. Through this process, the message may be modified, improved and added a convenient feature by many users. But we must detect the malicious attackers and prevent the malicious code from damaging the receiver or prevent the attackers from obtaining the private messages. Therefore the concept of multi-signcryption was proposed in[9] which can meet with the requirement of multi-signers performing together the signcryption operation on messages and a specific scheme called Seo-Lee scheme was proposed in [10]. It efficiently provides message flexibility, order flexibility, message verifiability, order verifiability, message confidentiality, message unforgeability, non-repudiation and robustness. Based on Seo-Lee scheme, a Multi-signcryption scheme using identity and bilinear pairing was proposed. It greatly decreases the cost of building and managing public key infrastructures ; the expense of the users' management of public-key and their certificates is avoided. Up to the present, various studies on ID-Based multi-signcryption have been proposed[12,13].

Most of the existing ID-Based multi-signcryption scheme don't provide the public verifiability though it is a very important property in many practical application. Even there are some schemes can provide the verification, they need the plaintext or the private key of verifier. In this paper, we propose a new ID-Based multi-signcryption that can provide public verification together with other security properties fulfilled and we also give the analysis of the scheme. 


\section{Preliminary Works}

\subsection{Bilinear Pairings}

We consider two groups $\mathrm{G}_{1}$ (additive) and $\mathrm{G}_{2}$ (multiplicative) of the same prime order $\mathrm{q}$. We need bilinear maps $\mathrm{e}: \mathrm{G}_{1} \times \mathrm{G}_{1} \rightarrow \mathrm{G}_{2}$ satisfying the following properties:

1. Bilinearity: $\forall \mathrm{P}, \mathrm{Q} \in \mathrm{G}_{1}, \forall \mathrm{a}, \mathrm{b} \in \mathrm{F}_{\mathrm{q}}^{*}$, we have $\mathrm{e}(\mathrm{aP}, \mathrm{bQ})=\mathrm{e}(\mathrm{P}, \mathrm{Q})^{\mathrm{ab}}$.

2. Non-degeneracy: The map does not send all pairs in $\mathbf{G}_{1} \times \mathbf{G}_{1}$ to the identity in G2. Observe that since $\mathrm{G}_{1}, \mathrm{G} 2$ are groups of prime order this implies that if $\mathrm{P}$ is a generator of $\mathrm{G} 1$ then $\mathrm{e}(\mathrm{P}, \mathrm{P})$ is a generator of $\mathrm{G} 2$.

3. Computability: there exists an efficient algorithm to compute $\mathrm{e}(\mathrm{P}, \mathrm{Q}) \forall \mathrm{P}, \mathrm{Q} \in \mathrm{G}_{1}$.

There are two problems that our scheme base on as follows:

DBDHP: Given two groups $\mathrm{G}_{1}$ and $\mathrm{G}_{2}$ of the same prime order q, a bilinear mape: $G_{1} \times G_{1} \rightarrow G_{2}$ and a generator $P$ of $G_{1}$ the Decisional Bilinear Diffie-Hellman problem (DBDHP) in $\left(G_{1}, G_{2}, e\right)$ is to decide whetherh $=\mathrm{e}(\mathrm{p}, \mathrm{p})^{\mathrm{abc}}$ given $(\mathrm{P}, \mathrm{aP}, \mathrm{bP}, \mathrm{cP})$ and an element $h \in G_{2}$.

CBDHP: Given two groups $\mathrm{G}_{1}$ and $\mathrm{G}_{2}$ of the same prime order q, a bilinear map $e: G_{1} \times G_{1} \rightarrow G_{2}$ and a generator $P$ ofG 1 , the Computational Bilinear Diffie-Hellman problem (CBDHP) in $\left(\mathrm{G}_{1}, \mathrm{G}_{2}, \mathrm{e}\right)$ is to compute $\mathrm{h}=\mathrm{e}(\mathrm{p}, \mathrm{p})^{\mathrm{abc}} \operatorname{given}(\mathrm{P}, \mathrm{aP}, \mathrm{bP}, \mathrm{cP})$.

No algorithm is known to be able to solve any of them so far, though DBDHP is no harder than CBDHP.

\subsection{Security properties}

Due to the identity-based nature of singncryption, and the combined requirements on confidentiality and non-repudiation, the security requirements are multifaceted and quite stringent. We assume Alice is the recipient, $I_{i}$ are the signers and Charlie is a third party. The properties a multi-signctyption should meets are as follows:

Confidentiality: It is impossible for the attacker to compute the secret messages $\mathrm{m}_{1}, \mathrm{~m}_{2}, \cdots, \mathrm{m}_{\mathrm{n}}$, or compute the private information of Alice by the signcryption .

Unforgeability: It is impossible for any attacker to forge a valid multi-signcryption even any one of $\mathrm{I}_{\mathrm{i}}$ or Alice.

Non-repudiation: Charlie can judge the validity of a signcryption when dispute occurs for sender and recipient.

\section{3 a new ID-Based multi-signcryption}

This section proposes a new ID-Based multi-signcryption schemes with flexibility and verifiability for both message and order.

\section{Setup}

The PKG chooses the system parameters that include two groups $\left(G_{1},+\right)$ and $\left(G_{2}, \cdot\right)$, a bilinear map $e: G_{1} \times G_{1} \rightarrow G_{2}$ between these groups, a generator $\mathrm{P}$ of $\mathrm{G} 1$, a master secret $s \in Z_{q}{ }^{*}$, and a public key $P p u b=s P \in G_{1}$. It also chooses a secure symmetric scheme (E;D) and hash 
functions $, H_{0}:\{0,1\}^{*} \rightarrow Z_{q}^{*}, H_{1}:\{0,1\}^{*} \rightarrow G_{1}$ and $H_{2}:\{0,1\}^{*} \times\{0,1\}^{n} \times G_{1} \rightarrow Z_{q}^{*}, \mathrm{n}$ is the length of plaintext. The public key and private key of Alice is $Q_{a}=H_{0}\left(I D_{a}\right), S_{a}=s Q_{a}$, the keys of Ii $(1 \leq \mathrm{i} \leq \mathrm{n})$ is $Q_{i}=H_{0}\left(I D_{i}\right), \quad S_{i}=s Q_{i}$. PKG keeps the s, the public parameters are $\mathrm{G}_{1}$, $\mathrm{G}_{2}, \mathrm{P}, \mathrm{P}_{\mathrm{Pub}}, \mathrm{e}, \mathrm{H}_{0}, \mathrm{H}_{1}, \mathrm{H}_{2}$.

\section{Signcrypt}

We assume that Alice wants $n$ signers Ii $(1 \leq \mathrm{i} \leq \mathrm{n})$ to generate a signcryption on a fixed message $M$ according to order fixed beforehand. First Alice send the original message $\mathrm{m}$ and the public key $Q_{a}=H_{0}\left(I D_{a}\right)$ to all the signers $\mathrm{Ii}(1 \leq \mathrm{i} \leq \mathrm{n})$, each signer changes the message $\mathrm{m}$ into $\mathrm{m}_{\mathrm{i}}$ which includes the secret information for Alice.

Assume $I_{1}$ is the first signer and $I_{i+1}$ is the next one to $I_{i}$.

$\mathrm{I}_{1}$ : choose $k_{1} \in Z_{q}^{*}$ in random, compute

$$
\begin{aligned}
& K_{1}=e\left(P_{p u b}, Q_{a}\right)^{k_{1}}, \quad r_{1}=H_{1}\left(m_{1}\left\|Q_{1}\right\| K_{1}\right), \quad c_{1}=E_{K_{1}}\left(m_{1} \| \mathrm{Q}_{1}\right), s_{1}=\left(k_{1} P_{p u b}-r_{1} S_{1}\right), \\
& X_{1}=k_{1} P, \quad R_{1}=H_{2}\left(I D_{1}, c_{1}, X_{1}\right) P_{p u b}, \quad Z_{1}=S_{1}+k_{1} R_{1}
\end{aligned}
$$

$\mathrm{I}_{1}$ sends $\left(Q_{1}, s_{1}, r_{1}, c_{1}, X_{1}\right)$ to $\mathrm{I}_{2}$, and sends $\mathrm{Z}_{1}$ to Alice.

According to each signer $\mathrm{I}_{\mathrm{i}}$.the signcryption process is as follows:

$\mathrm{I}_{\mathrm{i}}$ : receive $\left(Q_{i-1}, s_{i-1}, r_{i-1}, c_{i-1}, X_{i-1}\right)$, choose $k_{i} \in Z_{q}^{*}$ in random, compute:

$$
\begin{aligned}
& K_{i}=e\left(P_{p u b}, Q_{a}\right)^{k_{i}}, \quad r_{i}=H_{1}\left(m_{i}\left\|Q_{i}\right\| K_{i}\right) \cdot r_{i-1} \quad, \quad c_{i}=E_{K_{i}}\left(m_{i}\left\|\mathrm{Q}_{\mathrm{i}}\right\| Q_{i-1}\left\|s_{i-1}\right\| c_{i-1}\right), \\
& s_{i}=\left(k_{i} P_{p u b}-r_{i} S_{i}\right) \in G_{1}, \quad X_{i}=H_{1}\left(c_{i}, Q_{i}, K_{i}\right) X_{i-1}
\end{aligned}
$$

And sends $\left(Q_{i}, s_{i}, r_{i}, c_{i}, X_{i}\right)$ to the next signer $\mathrm{I}_{\mathrm{i}+1}$. Until the last signer $\mathrm{I}_{\mathrm{n}} \operatorname{sends}\left(Q_{n}, s_{n}, r_{n}, c_{n}, X_{n}\right)$ to Alice, Alice take the steps as follow to unsigncrypt the multi-signctyption and verify.

\section{Unsigncrypt}

Alice :

Receives $\left(Q_{n}, s_{n}, r_{n}, c_{n}, X_{n}\right)$, with the private key $\mathrm{Sa}$ to compute the session key with $\mathrm{I}_{\mathrm{n}}: K_{n}=e\left(s_{n}, Q_{a}\right) e\left(Q_{n}, S_{a}\right) r_{n}$,

With $m_{n}\left\|Q_{n}\right\| Q_{n-1}\left\|s_{n-1}\right\| c_{n-1}=D_{K_{n}}\left(c_{n}\right)$, get message $\mathrm{m}_{\mathrm{n}}, \mathrm{Q}_{\mathrm{n}}, \mathrm{Q}_{\mathrm{n}-} 1 \quad, \quad \mathrm{~s}_{\mathrm{n}-} 1 \quad, \mathrm{c}_{\mathrm{n}-}$ 1 ;from $r_{n-1}=H_{1}\left(m_{n}\left\|Q_{n}\right\| K_{n}\right)^{-1} r_{n}$ to recover $\mathrm{r}_{\mathrm{n}-1} \quad$,from $X_{n-1}=H_{1}\left(c_{n}\left\|Q_{n}\right\| K_{n}\right)^{-1} X_{n}$ to recover $\mathrm{X}_{\mathrm{n}-1}$. Then Alice computes each session key $K_{i}=e\left(s_{i}, Q_{a}\right) e\left(Q_{i}, S_{a}\right) r_{i}$ with $\mathrm{I}_{\mathrm{i}}, 1 \leq i \leq n$, get all the part signcryption $\left(Q_{i}, s_{i}, r_{i}, c_{i}, X_{i}\right)$ and message $\mathrm{m}_{\mathrm{i}}$ together with the order of signer. 
Do the same steps until Alice compute $X_{1}=H_{1}\left(c_{2}\left\|Q_{2}\right\| K_{2}\right)^{-1} X_{2}, r_{1}=H\left(m_{2}\left\|Q_{2}\right\| K_{2}\right)^{-1} r_{2}$, and unsigncrypt $\mathrm{m}_{1}, \mathrm{Q}_{1}$ through $\mathrm{K}_{1}$.

Finally, Alice accepts the signcryption if $r_{1}=H\left(m_{1}\left\|Q_{1}\right\| K_{1}\right)$ and $e\left(P, Z_{1}\right)=e\left(P_{p u b}, Q_{1}\right) e\left(X_{1}, R_{1}\right)$ are established, else refuses it.

\section{Public verify:}

If a signer $\mathrm{I}_{\mathrm{i}}$ denies the signcryption, Alice sends $\left(c_{1}, X_{1}, Z_{1}\right)$ to the third party to arbitrate.

The third party compute $R_{1}=H_{2}\left(I D_{1}, c_{1}, X_{1}\right) P_{p u b}$,judge the sincryption is true if $e\left(P, Z_{1}\right)=e\left(P_{p u b}, Q_{1}\right) e\left(X_{1}, R_{1}\right)$ is established.

Proof of correctness

$e\left(P, Z_{1}\right)=e\left(P, S_{1}+k_{1} R_{1}\right)=e\left(P, s Q_{1}+k_{1} R_{1}\right)=e\left(P, s Q_{1}\right) e\left(k_{1} P, R_{1}\right)=e\left(P_{p u b}, Q_{1}\right) e\left(X_{1}, R_{1}\right)$

\section{4 validity and security analysis}

Message flexibility: A message does not need to be fixed beforehand. Therefore each signer can modify an original message.

Order flexibility: Neither order of signers nor signers themselves need to be designated beforehand. Therefore we can easily change order of signers, add a new signer and exclude a signer.

Confidentiality: It is impossible for the attacker to compute the secret messages $\mathrm{m}_{1}, \mathrm{~m}_{2}, \ldots, \mathrm{m}_{\mathrm{n}}$, from the signcryptions $\left(Q_{i}, s_{i}, r_{i}, c_{i}, X_{i}\right)$ without $\mathbf{k}_{\mathrm{i}}, \mathbf{s}_{\mathrm{j}}$ and $\mathbf{s}_{\mathrm{q}}$. Base on the problem of discrete logarithm, it is also computational impossible for attacker to compute $\mathbf{k}_{\mathrm{j}}$ from $K_{i}=e\left(P_{p u b}, Q_{a}\right)^{k_{i}}$ or $\mathbf{\$}_{\mathrm{i}}$ from $s_{i}=\left(k_{i} P_{p u b}-r_{i} S_{i}\right)$. Therefore it is impossible to get $\mathrm{m}_{\mathrm{j}}$.

Unforgeability: It is impossible for any attacker to forge a valid signcryption without $\mathbf{S}_{\mathrm{i}}$, the private key of $\mathrm{I}_{\mathrm{i}}$ even any one of $\mathrm{I}_{\mathrm{i}}$ or Alice.

Non-repudiation: Since the signcryption of each $I_{\mathrm{i}}$ is unforgeable, once the multi-signcryption is generated, it cannot be denied.

Public verification: When dispute occurs for sender and recipient, the recipient can send $\left(c_{1}, X_{1}, Z_{1}\right)$ to the judge. The judge can verify the validity of the signcryption without the private key of recipient. And the information still kept secret since the verify uses cipher text. The judge first compute $R_{1}=H_{2}\left(I D_{1}, c_{1}, X_{1}\right) P_{p u b}$, then judge the sincryption is true if $e\left(P, Z_{1}\right)=e\left(P_{p u b}, Q_{1}\right) e\left(X_{1}, R_{1}\right)$ is established.

Assume that an attacker or Alice choose $k_{1}^{\prime} \in Z_{q}^{*}$ in random, compute $X_{1}^{\prime}=k_{1}^{\prime} P$, $R_{1}^{\prime}=H_{2}\left(I D_{1}, c_{1}^{\prime}, X_{1}^{\prime}\right) P_{p u b}, Z_{1}^{\prime}=S_{1}^{\prime}+k_{1}^{\prime} R_{1}^{\prime}$, forge a message $\left(c_{1}^{\prime}, X_{1}^{\prime}, Z_{1}^{\prime}\right)$, if $e\left(P, Z_{1}^{\prime}\right)=e\left(P_{p u b}, Q_{1}\right) e\left(X_{1}^{\prime}, R_{1}\right)$ established, namely 


$$
e\left(P, Z_{1}^{\prime}\right)=e\left(P, S_{1}^{\prime}+k_{1}^{\prime} R_{1}^{\prime}\right)=e\left(P, s^{\prime} Q_{1}+k_{1}^{\prime} R_{1}^{\prime}\right)=e\left(P, s Q_{1}\right) e\left(k_{1} P, R_{1}\right)=e\left(P_{p u b}, Q_{1}\right) e\left(X_{1}, R_{1}\right)
$$

Because the attacker doesn't know value of $\mathrm{s}$, if $e\left(P, s^{\prime} Q_{1}+k_{1}^{\prime} R_{1}^{\prime}\right)=e\left(P, s Q_{1}\right) e\left(k_{1} P, R_{1}\right)$ is satisfied, the problems of ECDL and BDH are solvable.

Verification flexibility: The public verification is alternative and calculates of $\mathrm{X}_{\mathrm{i}}$ can be omitted. Therefore it will be computed only when we need public verification.

\section{Conclusions}

This paper proposed a new ID-Based multi-signcryption with its analysis. The scheme can provides the public verification of signcryption and the signer. It also satisfies the security properties efficiently with short cipher text. It can be applied in e-commerce or e-voting.

\section{Supported by:}

Fundamental Research Funds for the Central Universities ( No. SWJTU09BR119). National Natural Science Foundation of China (Grant No.61003245)

\section{REFERENCES}

[1] Shamir A. Identity-based Cryptosystems and Signature Schemes [ C]//Advances in Cryptology, Crypto'84 : LNCS 196.New York : Springer Verlag, 1984 : 47-53.

[2] Boneh D, Franklin M. Identity-based encryption from the weil pairing[C]// Kilian J, ed. Advances in Cryptology-CRYPTO 2001, LNCS 2139. Berlin, Heidelberg: Springer-Verlag, 2001:213- 229.

[3] ZHEN G Y. Digital signcryption or how to achieve cost ( signature \&encryption) $<<\operatorname{cost}$ ( signature) + cost ( encryption ) [ M ]// KAL ISKI B. A dvances in Cry ptolog y : CRYPTO 1997 , Berlin : Springer ,1997 : 165-179.

[4] Ma C, Chen K. publicly verifiable authenticated encryption[J].Electronics Letters, 2003; 39( 3) : 281 282

[5]. Wang G L, Bao F, Ma C S . Efficient Authenticated Encryption Schemes with Public Verifiability[C].In: Proc of the 60th IEEE Vehicular Technology Conference (VTC 2004- Fall) IEEE Computer Society, 2004.

[6]Chan W K, Wei V K. A Threshold Proxy Signcryption [C]. Proc. Of International Conference on Security and Management, Monte Carlo Resort, Las Vegas, Nevada, USA, 2002: 24-27.

[7 ] Peng Y Q, Xie S Y, Chen Y F et al. A Publicly Verifiable Authenticated Encryption Scheme with Message Linkages.ICCNMC 2005, LNCS 3619, springer- Verlag Berlin Heidelberg, 2005 : $1271 \sim 1276$

[8 ] Dent Alexander W. Hybrid signcryption schemes wit h insider security. In : Proceedings of $t$ he Information Security and Privacy ACISP 2005 , Brisbane, Aust ralia , 2005, 253 266.

[9]Mitomi S , Miyaji A. A General Model of Multisignature Schemes with Message Flexibility, Order Flexibility, and Order Verifiability [J ]. IEICE Trans on Fundamentals , 2001 , E842A(10):88-99. 
[10] Seo S H, Lee S H.. A Secure and Flexible Multi-signcryption Scheme [C]/ / ICCSA 2004 : LNCS 3 046. Berlin : Springer Verlag, 2004 : 689-697.

[11]Zhang C R,Xiao G Z. Multi2signcryption scheme using identity and bil inear pairing [J]. JOURNAL OF XID IAN UNIVERSITY, 2007, 34(2):270-274 (in Chinese).

[12] Zhang J H,Wang J L, Wang Y M. A multi-signcryption model and its application [J] - Journal of Xidian University(Natural Science 2004(3) (in Chinese).

[13] Meng T, Zhang X P,Sun S H.identity-Based Multi-Sincryption scheme[J]. Journal of Electronics,2007,35(6):115-117 (in Chinese).

[14] Duan S, Cao Z. Efficient and Provably Secure Multi-receiver Identity-based Signcryption[C]//Proc. of Australasian Conference on Information Security and Privacy. [S. 1.]: Springer-Verlag, 2006: 195-206.

[15] Yu Yong, Yang Bo, Huang Xinyi, et al. Efficient Identity-based Signcryption Scheme for Multiple Receivers[C]//Proc. of ATC'07. [S. 1.]: Springer-Verlag, 2007. 